\title{
Bulk and Confined Benzene-Cyclohexane Mixtures Studied by an Integrated Total Neutron Scattering and NMR Method
}

\author{
Terri-Louise Hughes ${ }^{1}\left(\mathbb{D} \cdot\right.$ Marta Falkowska $^{1}\left(\right.$ Markus Leutzsch $^{2,4}\left(\right.$ M $^{-} \cdot$ Andrew J. Sederman $^{2}\left(\mathbb{D} \cdot\right.$ Mick D. Mantle $^{2}$.

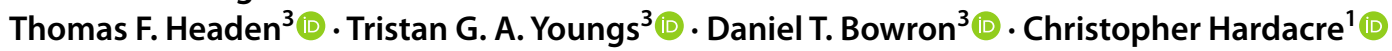

Accepted: 10 April 2021 / Published online: 23 April 2021

(c) Crown 2021

\begin{abstract}
Herein mixtures of cyclohexane and benzene have been investigated in both the bulk liquid phase and when confined in MCM-41 mesopores. The bulk mixtures have been studied using total neutron scattering (TNS), and the confined mixtures have been studied by a new flow-utilising, integrated TNS and NMR system (Flow NeuNMR), all systems have been analysed using empirical potential structure refinement (EPSR). The Flow NeuNMR setup provided precise time-resolved chemical sample composition through NMR, overcoming the difficulties of ensuring compositional consistency for computational simulation of data ordinarily found in TNS experiments of changing chemical composition-such as chemical reactions. Unique to the liquid mixtures, perpendicularly oriented benzene molecules have been found at short distances from the cyclohexane rings in the regions perpendicular to the carbon-carbon bonds. Upon confinement of the hydrocarbon mixtures, a stronger parallel orientational preference of unlike molecular dimers, at short distances, has been found. At longer first coordination shell distances, the like benzene molecular spatial organisation within the mixture has also found to be altered upon confinement.
\end{abstract}

Keywords Catalysis $\cdot$ EPSR $\cdot$ TNS $\cdot$ NMR $\cdot$ Confinement

\section{Introduction}

Modern day living is increasingly reliant on heterogeneously catalysed processes, of which increased understanding is critical [1]. The confinement of liquids in solid materials is abundant in the field, where reactions proceed within the pores of solid materials. Liquids confined in mesoporous

Terri-Louise Hughes

terri-louise.hughes@manchester.ac.uk

Marta Falkowska

marta.falkowska@manchester.ac.uk

1 Department of Chemical Engineering and Analytical Science, The University of Manchester, Manchester M13 9PL, UK

2 Department of Chemical Engineering and Biotechnology, University of Cambridge, Philippa Fawcett Drive, Cambridge CB3 OAS, UK

3 STFC ISIS Facility, Rutherford Appleton Laboratory Chilton, Didcot OX1 10QX, Oxon, UK

4 Present Address: Max-Planck-Institut Für Kohlenforschung, 45470 Mülheim/Ruhr, Germany materials, those with pore sizes between $20 \AA$ and $500 \AA$, have been shown to display altered physical and thermodynamic properties in comparison to that of the bulk liquids. The least complex of the confined liquid systems, that of confined pure water, has been the subject of multiple studies, with changes in density and surface tension identified as well as altered phase change transition temperatures of interfacial water molecules [2,3]. A recent review by Liu and Zhang has highlighted many properties of hydrocarbons influenced by confinement, including but not limited to the density profiles, the surface tension, fluid viscosity and fluid mobility of the liquids [4]. Structural orientation and organisation of benzene molecules are impacted by confinement, as detailed by Falkowska et al., when using total neutron scattering (TNS) and empirical potential structure refinement (EPSR) to study benzene confined in MCM-41 [5]. In contrast to that of single liquids, the understanding of more complex confined mixture systems is limited. In bulk systems tert-butanol and toluene are fully miscible, however Hamid et al. observed microphase separation of the two components when under confinement [6], with a central toluene rich core and tert-butanol forming an outer shell at 
the pore surface. It is important that the confinement effects of mixtures are further understood, with major emphasis on the impact of these properties on changes to reactivity.

TNS is a valuable technique which allows the study of a variety of systems. The scattering pattern obtained from a molecular liquid correlates to a complex sum of many structural correlations detailing the atomic pair distribution contributions. Due to this complexity, computer models are used to simulate and analyse the molecular liquid structure. Using these methods the structure of several organic solvents, including benzene and cyclohexane, have been studied previously [7, 8]. The EPSR modelling software was used to simulate the bulk liquids, and excellent agreement between the data and the models was achieved. More recently, an investigation of the hydrogenation of benzene to cyclohexane using a new integrated TNS and NMR technique, NeuNMR has been reported [9]. The inclusion of NMR enabled time-resolved chemical composition data to be obtained simultaneously with the TNS data. Obtaining simultaneous data on the chemical speciation is of significant use in both the data correction and data analysis processes, particularly in the case of reacting systems. The most significant disadvantage to this integrated system was the short data acquisition times that were necessarily employed, coupled with the modest strength of the NMR magnet.

Herein, an integrated TNS and NMR technique utilising flow conditions (Flow NeuNMR) has been developed. This system can theoretically be studied for an infinite length of time, with longer acquisition times resulting in improved statistics and better signal to noise ratios in the attained data. This in turn results in improved reliability of models derived through post data collection simulation/modelling. The mixed benzene-cyclohexane system confined in MCM-41 has been studied through use of this innovative setup. In order to investigate the influence of confinement on molecular interactions in benzene-cyclohexane mixtures, the bulk phase mixtures of these two components have also been studied using typical TNS experimental procedures. The mixed benzene and cyclohexane system has been chosen as the target due to the high contrast between scattering length density of the respective hydrocarbons, particularly when confined in MCM-41 [10]. An active catalyst was selected in the design of this experiment, to probe the possibility of establishing a mixture stoichiometry under reaction conditions using the flow system.

EPSR was used to simulate and investigate the bulk and confined mixture systems. EPSR derives a 3D model structure that is consistent with experimental data, starting from known chemical and physical restraints and a reference interatomic potential. An additional interatomic potential is then added and iteratively adjusted until the best agreement possible with the experimental diffraction data is obtained. After the simulated model fits the data acceptably, the model can then be probed for structural properties of the system of interest.

\section{Methods}

\subsection{Experimental}

Cyclohexane- $h_{12}$, benzene- $h_{6}$, cyclohexane- $d_{12}$ and benzene$d_{6}$, were purchased from Sigma-Aldrich, now Merck KGaA, as was the MCM-41 catalyst support. Platinum (IV) nitrate solution, Pt 15\% w/w was purchased from Alfa- Aesar.

All experiments were performed using the Near- and InterMediate-Range Order Diffractometer (NIMROD) at the ISIS facility at the STFC Rutherford Appleton Laboratory, Harwell Campus, Oxfordshire, Oxford, UK [11].

$\mathrm{F}(\mathrm{Q})=\sum_{\alpha \beta \geq \alpha}\left(2-\delta_{\alpha \beta}\right) \mathrm{c} \alpha \mathrm{c} \beta \mathrm{b} \alpha \mathrm{b} \beta \mathrm{S} \alpha \beta(\mathrm{Q})$

Equation 1 details the total structure factor $\mathrm{F}(Q)$ obtained in neutron scattering experiments. The differential cross section is measured as a function of magnitude of the momentum vector $Q$. This contains contributions from both single atom scattering and correlations between atom pairs. Each interaction between pairs of atoms in a sample, denoted to as partial structure factors $\mathrm{S}_{\alpha \beta}(Q)$, are weighed by the concentrations $c_{\alpha}, c_{\beta}$ and the atomic scattering lengths $b_{\alpha}, b_{\beta}$ for atoms $\alpha$ and $\beta$. This is expressed as the sum of all correlations, with the Kronecker delta $\delta_{\alpha \beta}$ used to avoid double counting atom pair terms.

\subsubsection{Bulk Mixtures}

Benzene-cyclohexane mixed bulk systems have been measured at molar ratios of, 3:1, 1:1 and 1:3. To exploit the isotopic contrast between hydrogen and deuterium, multiple isotopic compositions were collected for each cyclohexanebenzene mixture studied. TNS data of the fully protiated system, the fully deuterated system, an equimolar protiated to deuterated system, alongside several other isotopic mixtures were collected, all are detailed in Table 1.

For each sample, scattering data was accumulated for a minimum of $40 \mu \mathrm{A}$, corresponding to approximately $1 \mathrm{~h}$ of counting time per sample. Scattering measurements of the empty sample cells, the empty diffractometer and a $3.0 \mathrm{~mm}$ thick vanadium standard sample were collected for data correction and normalisation. Measurements were taken at a maintained temperature of $298 \pm 0.1 \mathrm{~K}$. For each bulk mixture measurement a total volume of $1.8 \mathrm{~mL}$ of liquid was used. The liquid mixtures were loaded into flat plate, null-scattering, $\mathrm{Ti}_{0.68} \mathrm{Zr}_{0.32}$ containers of internal dimensions $35 \times 40 \times 1 \mathrm{~mm}$, with a wall thickness of $1 \mathrm{~mm}$. 
Table 1 Isotopic substitutions of benzene and cyclohexane molecules used in the collection of bulk mixture TNS data

\begin{tabular}{|c|c|c|c|c|c|}
\hline \multicolumn{2}{|c|}{$\begin{array}{l}1: 3 \text { benzene-cyclohex- } \\
\text { ane bulk mixture } \\
\text { isotopic substitutions }\end{array}$} & \multicolumn{2}{|c|}{$\begin{array}{l}1: 1 \text { benzene-cyclohex- } \\
\text { ane bulk mixture } \\
\text { isotopic substitutions }\end{array}$} & \multicolumn{2}{|c|}{$\begin{array}{l}3: 1 \text { benzene- } \\
\text { cyclohexane } \\
\text { bulk mixture } \\
\text { isotopic substi- } \\
\text { tutions }\end{array}$} \\
\hline $\mathrm{h}_{6}$ & $\mathrm{~h}_{6}$ & $\mathrm{~h}_{6}$ & $\mathrm{~h}_{12}$ & $\mathrm{~h}_{6}$ & $\mathrm{~h}_{12}$ \\
\hline $\mathrm{d}_{6}$ & $\mathrm{~d}_{6}$ & $\mathrm{~d}_{6}$ & $\mathrm{~h}_{12}$ & $\mathrm{~d}_{6}$ & $\mathrm{~h}_{12}$ \\
\hline $\mathrm{h}_{3} \mathrm{~d}_{3}$ & $\mathrm{~h}_{3} \mathrm{~d}_{3}$ & $\mathrm{~h}_{3} \mathrm{~d}_{3}$ & $\mathrm{~h}_{6} \mathrm{~d}_{6}$ & $\mathrm{~h}_{3} \mathrm{~d}_{3}$ & $\mathrm{~h}_{6} \mathrm{~d}_{6}$ \\
\hline $\mathrm{d}_{6}$ & $\mathrm{~d}_{6}$ & $\mathrm{~d}_{6}$ & $\mathrm{~h}_{6} \mathrm{~d}_{6}$ & $\mathrm{~d}_{6}$ & $\mathrm{~h}_{6} \mathrm{~d}_{6}$ \\
\hline $\mathrm{h}_{6}$ & $\mathrm{~h}_{6}$ & $\mathrm{~h}_{6}$ & $\mathrm{~d}_{12}$ & $\mathrm{~h}_{6}$ & $\mathrm{~d}_{12}$ \\
\hline $\mathrm{h}_{3} \mathrm{~d}_{3}$ & $\mathrm{~h}_{3} \mathrm{~d}_{3}$ & $\mathrm{~h}_{3} \mathrm{~d}_{3}$ & $\mathrm{~d}_{12}$ & $\mathrm{~h}_{3} \mathrm{~d}_{3}$ & $\mathrm{~d}_{12}$ \\
\hline $\mathrm{d}_{6}$ & $\mathrm{~d}_{6}$ & $\mathrm{~d}_{6}$ & $\mathrm{~d}_{12}$ & $\mathrm{~d}_{6}$ & $\mathrm{~d}_{12}$ \\
\hline
\end{tabular}

Polytetraflouroethylene (PTFE) O-rings were used to seal the cells which were placed on an automatic sample changer.

\subsubsection{Confined Mixtures}

The $3 \mathrm{wt} \% \mathrm{Pt} / \mathrm{MCM}-41$ catalyst used in this study was prepared by the pore volume impregnation of the commercially sourced catalyst MCM-41 support [12]. At the ISIS facility, the catalyst was further heated to $453 \mathrm{~K}$ for $48 \mathrm{~h}$ in a vacuum oven, then immediately stored in an argon filled glove box. Any sample handling, including the filling of sample cans, was also undertaken in the glove box. A catalyst bed height of $4 \mathrm{~cm}$ was used for both the TNS and NMR samples, corresponding to $\sim 340 \mathrm{mg}$ and $\sim 170 \mathrm{mg}$ of material, respectively. The position of the sample beds was fixed using silica wool plugs. All investigations were performed without removal/replacement of the respective TNS and NMR catalyst samples.

Custom $6.35 \mathrm{~mm}$ outer diameter, $1 \mathrm{~mm}$ thickness, open ended vanadium tubes were used as the TNS sample cells. These were purchased from Metal Technology, Albany, USA. Long length, $400 \mathrm{~mm}$, open ended medium wall NMR tubes with $3.4 \mathrm{~mm}$ inner diameter and $0.8 \mathrm{~mm}$ thickness, were used as the NMR sample cells. These were purchased from Goss Scientific Instruments Ltd, Cheshire, UK. The neutron data was acquired using an $8 \times 30 \mathrm{~mm}(\mathrm{~W} \times \mathrm{H})$ beam size, with a time resolution of $5 \mathrm{~min}$ per scattering profile. A minimum of 12 data sets at a time were merged during the subsequent analysis, equating to $\geq 40 \mu \mathrm{A}$ of data accumulation and giving acceptable statistics on the data. ${ }^{1} \mathrm{H}$ NMR data has been acquired, with a one minute time resolution using a Pulsar $60 \mathrm{MHz}$ spectrometer (Oxford Instruments, UK) equipped with a ${ }^{1} \mathrm{H} /{ }^{13} \mathrm{C}$ probe with deuterium lock. A boron carbide plate was used on top of the spectrometer body in order to shield it from neutrons scattered from the sample, and to prevent any unwanted scattering from the spectrometer itself. The electronics necessary to drive the
Temperature controlled gas handling panel with liquid-vapour saturators

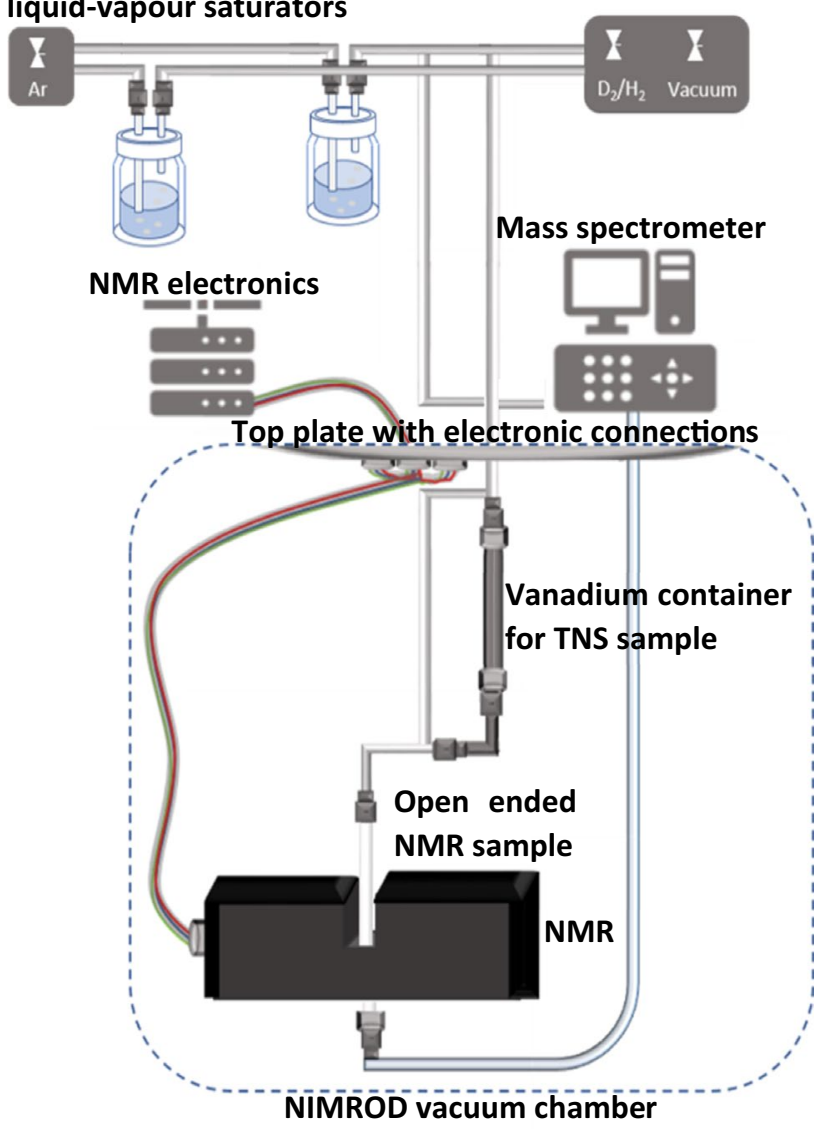

Fig. 1 Schematic of Flow Neu-NMR set-up used for the NeuNMR study of confined benzene: cyclohexane liquid mixtures

NMR spectrometer were connected via a vacuum tight topplate providing connectivity for the necessary power and data lines, and which was designed and provided by ISIS. Both sample cells were connected to a gas handling panel developed at ISIS via $1 / 4$ inch Ultra-Torr vacuum fittings. The integrated Flow Neu-NMR set-up is depicted in Fig. 1.

Two separate liquid mixing containers, each filled with a single hydrocarbon liquid have been utilised. These liquid mixing containers had a separate flow of carrier gas to enable varying ratios of the two deposited hydrocarbons. Online hydrocarbon mixture deposition was achieved by altering the flow of the respective hydrocarbon containing argon carrier gases, between 1 and $9 \mathrm{~mL} \mathrm{~min}{ }^{-1}$. A hydrogen/deuterium gas flow of $1 \mathrm{~mL} \mathrm{~min}{ }^{-1}$ was combined with the carrier gas/ hydrocarbon flow prior to passing through the catalyst feed. The NMR signal and the TNS structure factor showed that an equilibrated state had been achieved after approximately $15 \mathrm{~min}$ of carrier gas/hydrocarbon/hydrogenation gas flow.

Four flow mixtures have been studied, two fully protiated experiments and two intentionally identical corresponding fully deuterated experiments. These compositions, from this 
Table 2 Details of fully protiated experiments performed using the Flow Neu-NMR system

\begin{tabular}{llll}
\hline $\begin{array}{l}\text { Ar flow through } \\
\text { benzene- } \\
\text { min }_{6} \mathrm{~mL}\end{array}$ & $\begin{array}{l}\text { Ar flow through } \\
\text { cyclohexane- } \\
\text { min }^{-1}\end{array}$ & $\mathrm{H}_{2} \mathrm{~mL} \mathrm{~min}^{-1}$ \\
\hline $1-h$ & 6.7 & 2.3 & 1.0 \\
$2-h$ & 4.5 & 4.5 & 1.0 \\
\hline
\end{tabular}

Table 3 Details of fully protiated experiments performed using the Flow Neu-NMR system

\begin{tabular}{llll}
\hline & $\begin{array}{l}\text { Ar flow through } \\
\text { benzene- } \mathrm{d}_{6} \mathrm{~mL} \\
\min ^{-1}\end{array}$ & $\begin{array}{l}\text { Ar flow through } \\
\text { cyclohexane- } \mathrm{d}_{12} \mathrm{~mL} \\
\mathrm{~min}^{-1}\end{array}$ & $\mathrm{D}_{2} \mathrm{~mL} \mathrm{~min}^{-1}$ \\
\hline $1-d$ & 6.7 & 2.3 & 1.0 \\
$2-d$ & 4.5 & 4.5 & 1.0 \\
\hline
\end{tabular}

point forward be identified numerically with a suffix of - $h$ or $-d$ denoting the hydrogen isotope, as detailed in Tables 2 and 3. The fully deuterated flow experiments allow superior quality neutron data collection since the overall incoherent background contribution is minimised, whilst the fully protiated experiments allows the corresponding ${ }^{1} \mathrm{H}-\mathrm{NMR}$ data analysis. Complementary to the flow mixtures, a pure benzene- $h_{6}$ flow was studied. This standard enabled the detection and quantification of hydrogenation reactivity within the flow system.

\subsection{Computational Simulations}

The GudrunN software package was used for the normalisation and correction of the TNS data prior to use in EPSR [13].

\subsubsection{Bulk Liquids}

The bulk liquid EPSR refinements were initialised from an equilibrated Monte Carlo simulation at $298 \mathrm{~K}$ containing 400 molecules in a cubic box. Cubic box lengths of $38.32 \AA$, $40.28 \AA$ and $42.35 \AA$ have been used for the $1: 3,1: 1$ and $3: 1$ benzene-cyclohexane bulk mixtures respectively, equating to respective atomic densities of 0.0960 atoms $\AA^{-3}$, 0.0918 atoms $\AA^{-3}$ and 0.0869 atoms $\AA^{-3}$. For each bulk liquid hydrocarbon mixture, the TNS experimental data acquired from all isotopic compositions was input to the EPSR refinement.

\subsubsection{Confined Flow Mixtures}

Optimised empty pore models were constructed with a rhombic prism shaped box $\left(\gamma=120^{\circ}\right)$ with cell parameters $(\mathrm{A} \times \mathrm{B} \times \mathrm{C})$ of 42.0, 42.0, and 50.0 $\AA$ respectively, and a pore diameter of $31.5 \AA$. The use of this shaped box in EPSR accounts for the hexagonal arrangement of the pores in MCM-41 due to the periodic boundary conditions applied. The pore models were assembled of 786 silicon atoms, 1572 oxygen and 79 water molecules, equating to a 0.0694 atoms $\AA^{-3}$ pore wall density. The construction of the empty MCM41 pore follows the procedure outlined by Soper and Bowron [14], using artificial Q-atoms to build and maintain the pore structure.

Subsequently, the EPSR models of flow 1 and flow 2 have been constructed by inserting benzene- $d_{6}$ and cyclohexane$d_{12}$ molecules in stoichiometric proportions corresponding to the ${ }^{1} \mathrm{H}-\mathrm{NMR}$ calculated compositions. The amount of benzene and cyclohexane molecules inserted into the hydrocarbon type inserted into the respective models is as follows, corresponding to a total hydrocarbon density of 0.0808 atoms $\AA^{-3}$ [7]. The hydrocarbon-filled pore simulation of flow 1 comprises 213 benzene molecules and 33 cyclohexane molecules, the hydrocarbon-filled pore simulation of flow 2 comprises 156 benzene molecules and 71 cyclohexane molecules.

\section{Results and Discussion}

\subsection{NMR}

The ${ }^{1} \mathrm{H}-\mathrm{NMR}$ analysis of the reference pure benzene flow system detailed no significant product formation. The broad peaks obtained by the ${ }^{1} \mathrm{H}-\mathrm{NMR}$ analysis, as a feature of the analysis of liquids under confinement, result in a compositional error of $\pm 5 \%$. Therefore, the amount of benzene hydrogenated under the selected flow-rate conditions of this report is, if any, lower than 5\%. This level of conversion is below the detection limit of the TNS analysis, and thus the stoichiometric composition of the benzene-cyclohexane mixtures of this study is for all practical purposes entirely defined by carrier gas flow rates through the pure solvent reservoirs feeding the reaction cells. Effectively this means that the reaction media is replenished in pores of the platinum loaded catalyst bed faster than significant levels of benzene conversion can take place.

Figure 2 displays the ${ }^{1} \mathrm{H}-\mathrm{NMR}$ spectra obtained for experiments $1-h$ and $2-h$ respectively. Analysis of the data reveals an $87 \%$ benzene- $h_{6} 13 \%$ cyclohexane- $h_{12}$ mixture composition, as a result of flow $1-h$. A $69 \%$ benzene- $h_{6} 31 \%$ cyclohexane- $h_{12}$ mixture composition is found as a result of flow 2- $h$. Assuming an approximate equal uptake of hydrocarbons from the respective liquid reservoirs by the carrier gas, as would be expected based upon the similar vapour pressures of the molecular liquids [15, 16], a preferential absorbance of benzene over cyclohexane is observed by ${ }^{1} \mathrm{H}-\mathrm{NMR}$ in the catalyst pores in comparison with the 
Fig. 2 1H-NMR data collected from experiments $1-\mathrm{h}$ and $2-\mathrm{h}$. The black line shows the measured data, blue the fitted NMR peaks, magenta their sum and red the residual of the fitted and the measured data

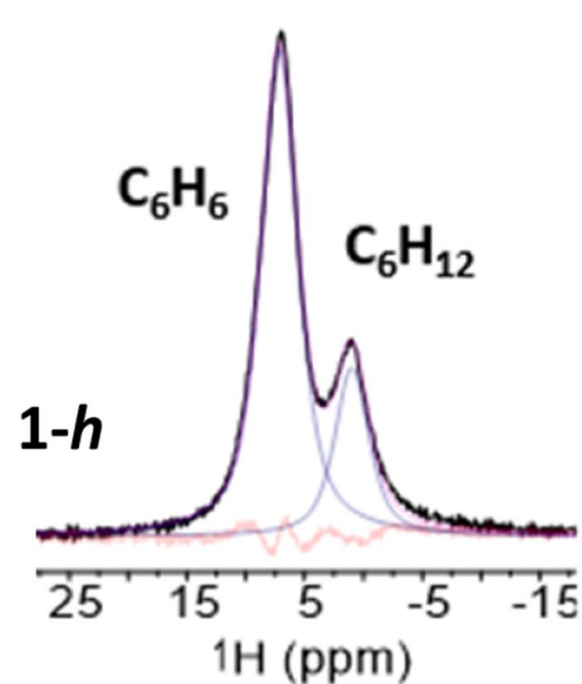

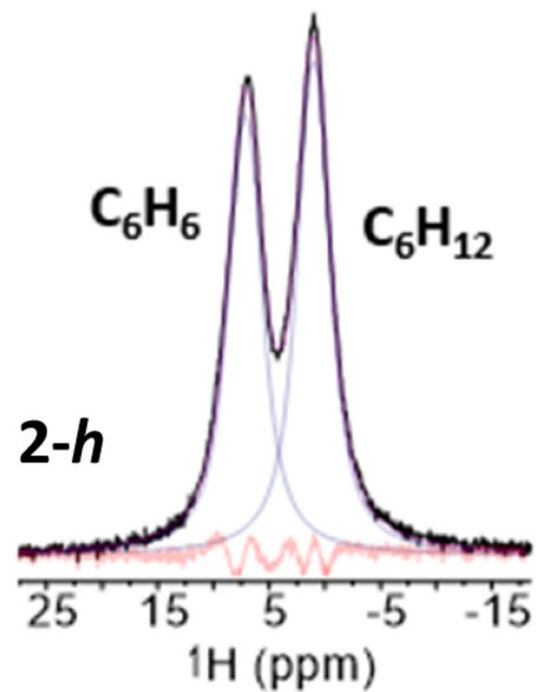

stoichiometry present within the inlet flows. The 1- $d$ and 2- $d$ TNS experimental data sets were used exclusively in the respective flow 1 and flow 2 EPSR refinements.

\subsection{Model Fits}

The wide $Q$ range accessed by NIMROD, herein optimised for a range of $0.05 \leq Q \leq 30 \AA^{-1}$, enables the collection of
TNS data that encompasses the atomistic and mesoscopic detailing regions. Figure 3 displays the experimental total structure factors and the simulated structure factors obtained for both the bulk liquid mixtures and the confined flow systems. The fits obtained for all bulk liquid mixtures simultaneously satisfy the data collected from the 7 corresponding isotopic mixes. Excellent agreement is shown in all cases. There is a minimal amount of disagreement at $Q$ values
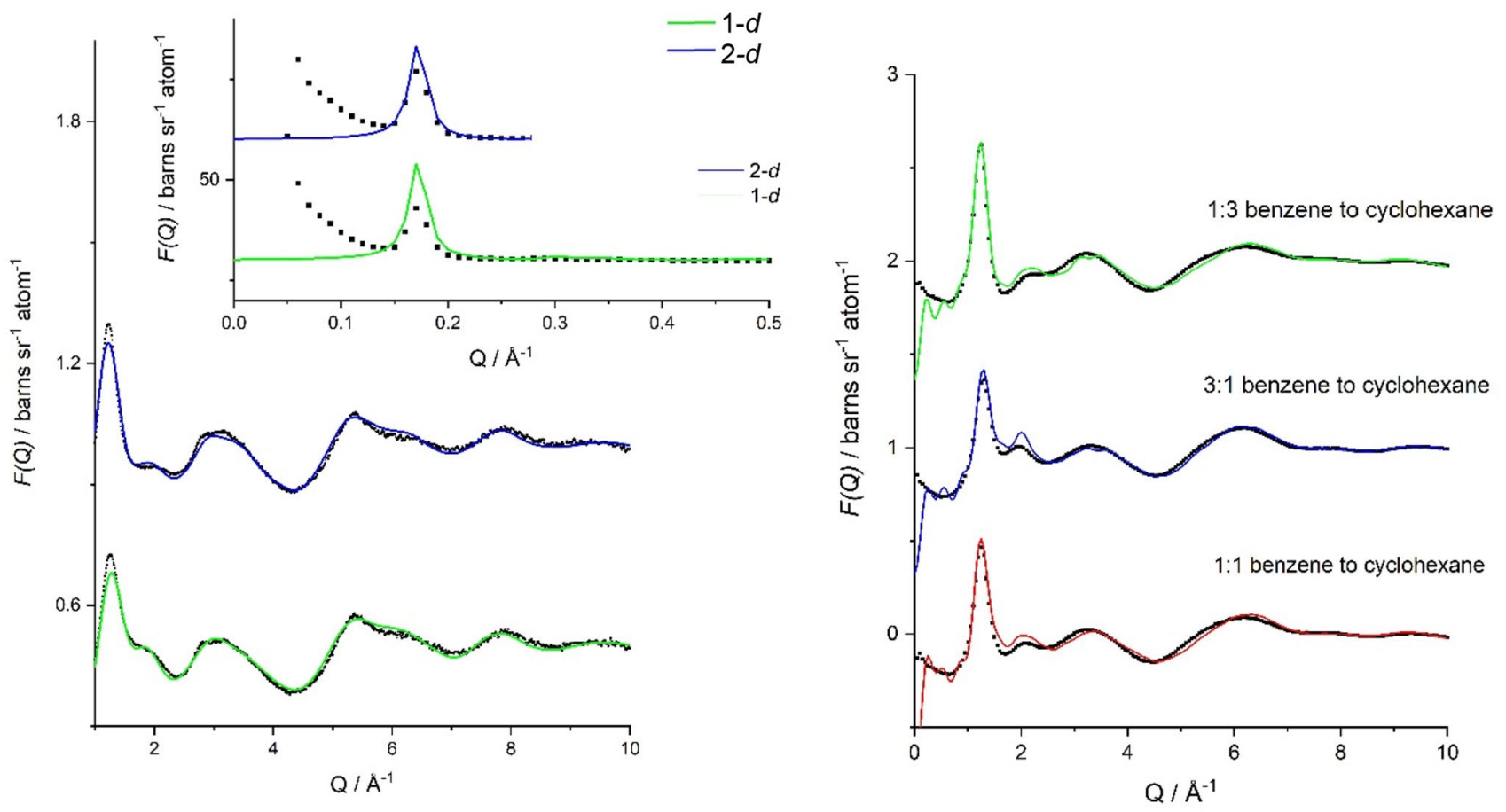

Fig. 3 Left-The experimental total structure factor (black) and the corresponding EPSR fitting data of the confined flow mixtures 1 (green) and 2 (blue). Right-The experimental total structure factor (black) and the corresponding EPSR fitting data of the benzenecyclohexane bulk mixtures of ratios 1:1 (red), 1:3 (green) and 3:1 (blue) 
lower than $0.8 \AA^{-1}$ caused by a small residual error in the inelasticity subtractions of the bulk system data, and in low- $Q$ region of the MCM- 41 confined mixtures from the Porod (particle surface and interface) scattering that is not accounted for in the model. In all cases the refinement of the models over the interatomic, intermolecular and poreconfined length scales is not significantly affected by these issues.

The higher $Q$ region, $1 \geq Q \geq 30 \AA^{-1}$, and the lower $Q$ region, $0.05 \geq Q \geq 0.5 \AA^{-1}$, are presented individually for the confined flow mixtures. The lower $Q$ region encompasses the Bragg features of the system, corresponding to the spacing of the mesopores. These experimental Bragg features are reproduced to a good extent by the simulations, with the primary Bragg (100) feature of flow 1 slightly overestimated. The higher $Q$ region contains the molecular and atomistic correlations, in both confined flows this data is reproduced with excellent agreement to the experimental data. The agreement demonstrates that the intramolecular and intermolecular correlations within the confined flow systems are well-described by the refined models.

\subsection{Radial Distribution Functions}

The radial distribution function (RDF) is used to describe the distribution of atoms as a function of distance from the central atom. This is obtained by performing a Fourier transform on the total structure factor, where $\rho$ is the atomic density, $r$ is the distance from the central atoms and $g_{\alpha \beta}(r)$ is a weighted sum of the pair distribution functions.

$$
\begin{aligned}
& \mathrm{G}(r)=\frac{1}{(2 \pi)^{3} \rho} \int_{0}^{\infty} \frac{4 \pi Q^{2} F(Q) \sin Q r}{Q r} d Q \\
& =\sum_{\alpha \beta \geq \alpha}\left(2-\delta_{\alpha \beta}\right) \operatorname{c} \alpha \mathrm{c} \beta \mathrm{b} \alpha \mathrm{b} \beta(\mathrm{g} \alpha \beta(\mathrm{r})-1)
\end{aligned}
$$

Herein, the coordinate output file of the simulated model allows for the calculation of radial distribution functions between the molecular centres of masses (COM-RDFs). The first species denoted in the molecular interactions is that of the central molecule, the second species type is that of the coordinating molecules. Figure 4 displays the COMRDFs, for like species and for molecules of unlike species, for the investigated cyclohexane-benzene mixtures. Comparison of the COM-RDFs, between bulk mixtures shows minimal deviation from one another. In all cases, the position of the $1^{\text {st }}$ coordination shell maximum is at a shorter distance, $\mathrm{g}(r) \sim 5.8 \AA$, for benzene-benzene correlations then for either the unlike or like cyclohexane including molecular correlations. The furthest 1 st coordination shell maximum is found for interactions between like cyclohexane molecules, $\mathrm{g}(r) \sim 6.2 \AA$. The 1 st coordination shell maximum for unlike molecules has been consistently calculated at a length, $g(r) \sim 6.1 \AA$, closer to that of like cyclohexane molecules than that of like benzene molecules. These findings are consistent with previously reported TNS studies of the respective pure liquids [7]. As displayed by the unlike molecular COM-RDFs, the coordination shell of cyclohexane molecules at distances $<4.5 \AA$ is primarily composed of benzene molecules. In line with the coordination composition of cyclohexane molecules at short distances, between $4.0 \AA$ and $5.0 \AA$, noted in the study of a simulated equimolar bulk cyclohexane-benzene mixture by Milano and MüllerPlathe [17].

In comparison to the bulk solvent mixtures, the COMRDFs of the confined mixed flow systems are perturbed. The cyclohexane-cyclohexane 1st coordination shell maxima are found at distances $g(r) \sim 6.2 \AA$ and $5.9 \AA$ for confined flows 1 and 2 , respectively. In the first instance this is equivalent to that of the analogous bulk mixture, whereas, in flow 2 this distance is $0.3 \AA$ shorter. The 1st coordination shell maxima of unlike molecular interactions for confined flows 1 and 2, calculated at lengths, $g(r) \sim 5.8$ and $6.0 \AA$ respectively, are both at slightly shorter lengths than found in the bulk mixtures. The $1^{\text {st }}$ coordination shell maximum for benzenebenzene correlations is constant between confined flows, $\mathrm{g}(r) \sim 6.0 \AA$, at comparatively longer distance than has found in the bulk mixtures.

The total number of 1 st shell coordination molecules for the bulk mixtures, varies between 12.0 and 13.1, which is in line with the reported data by Falkowska et al. [7] Headen et al. [18] and Milano et al. [17]. The lower total numbers of $1^{\text {st }}$ shell coordinated hydrocarbons found in the confined flow systems, between 9.1 and 10.8 , in comparison to the bulk systems is attributed to the 'large number of surface interactions between the silica and the confined liquid', as has been observed by Falkowska et al. [5], in the study of pure bulk and confined benzene. The percentage of each hydrocarbon species in the bulk mixture $1^{\text {st }}$ coordination shells, shows average deviations of $<1 \%$ from the overall proportion of the species present in the pore. Slightly increased average deviations of $\approx 3 \%$ have been observed for the confined systems. For both the bulk and confined mixture systems, irrespective of the central molecule type, the first coordination shell nearest neighbours are shown to be intrinsically proportional to the overall hydrocarbon ratio of the mixture.

\subsection{Angular Radial Distribution Functions}

The angular radial distribution functions (ARDF) describe the local orientational structure of molecules, providing access to orientation preferences. Herein, the ARDFs have been plotted as a function of the angle $\theta-m$ between the $z$-axis of the central molecule and the surrounding molecules 
Fig. 4 Radial distribution functions between the molecular centres of geometry for like cyclohexane molecules (red), like benzene molecules (blue) and dissimilar molecules (green), for all bulk hydrocarbon mixtures studied and confined flow systems

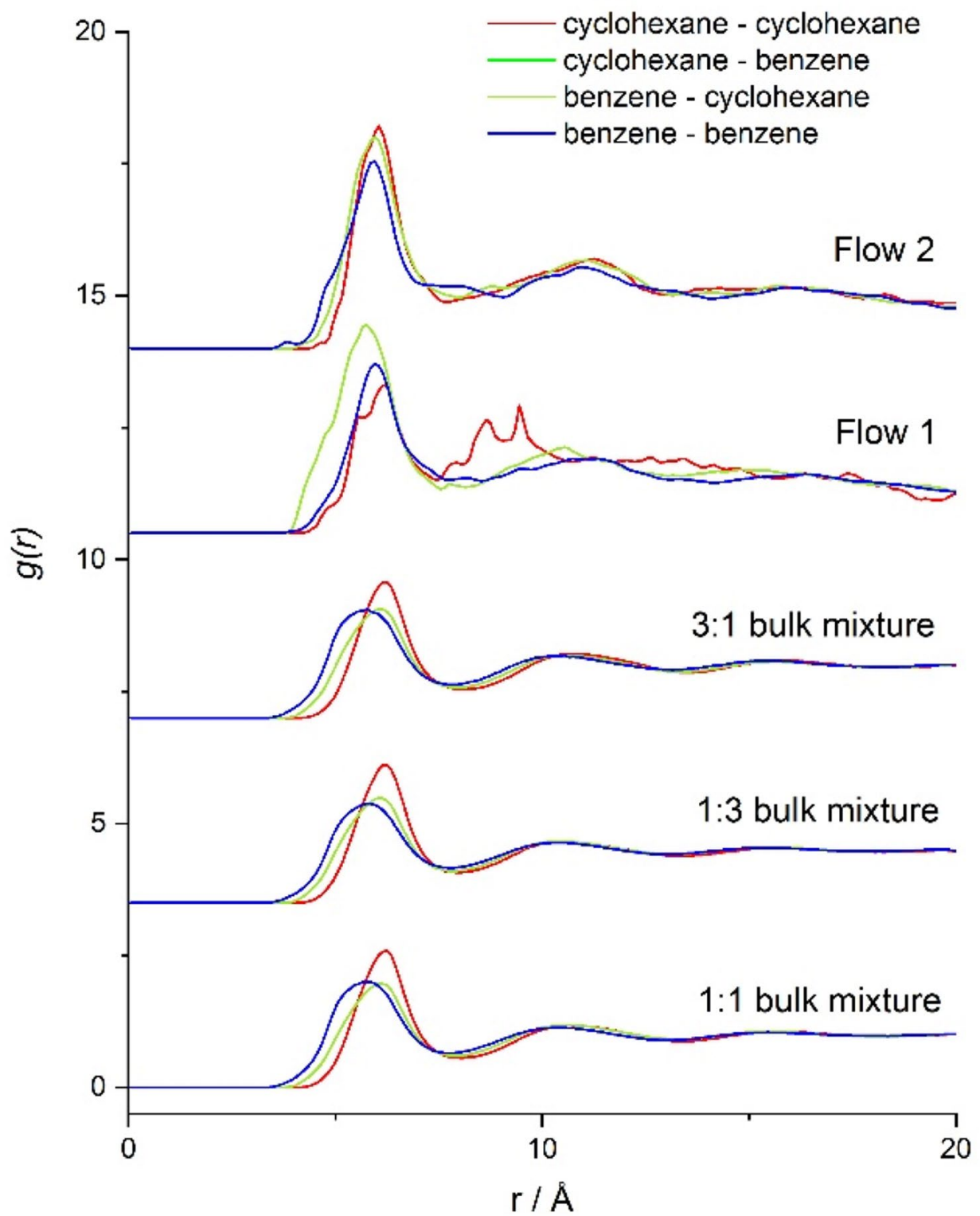

as defined from the surrounding molecular centre of mass $\left(0^{\circ} \leq \theta-m \leq 90^{\circ}\right)$, therefore, can be dictated as COM-ARDFs. Figure 5 displays the orientation of hydrocarbons, including the angle $\theta-m$ between the central and surrounding molecules, used to calculate the ARDFs. The $x$-axis and $y$-axis are defined in the plane of the ring, the $z$-axis is positioned out of the ring plane.

Shown in Fig. 6 are the calculated COM-ARDFs of all intermolecular correlations in the $1: 1$ bulk mixture, and in the confined flow systems 1 and 2 . All bulk mixtures studied displayed near identical COM-ARDFs, therefore, only one set has been exhibited as an example.

In the bulk mixtures, like cyclohexane molecules have an overall affinity for perpendicular orientations with a slight preference for parallel orientations at shorter length scales, $<4.95 \AA$, as observed in the form of a shoulder peak at shorter length scales. For like benzene molecules an increase in the prominence of this peak is displayed, indicating the preference of parallel arrangements at short lengths. For like benzene molecules perpendicular arrangements are observed above $4.95 \AA$. These like dimer results agree with the molecular arrangements of the respective pure liquids investigated by Falkowska et al. [7] and Headen et al. [18].

The unlike molecular COM-ARDFs display a distinct doublet spanning from 3.5 to $7.5 \AA$ at orientational values of $\theta-m=0 \pm 10^{\circ}$, this doublet transitions to a single sharp peak with maxima $5.95 \AA$ at values $\theta=90 \pm 10^{\circ}$. The intensities of these functions indicate, a preference for parallel orientated molecules at short distances, whilst perpendicular orientated molecules are observed together with parallel orientated 
Fig. 5 Definition of the angle $\theta$-m between the $z$-axis of the central reference molecule and the surrounding molecules used for the calculation of angular radial distribution functions, $\mathbf{b}$ Molecules oriented in parallel $\left(\theta-\mathrm{m}=0 \pm 10^{\circ}\right)$, c Molecules oriented perpendicularly $\left(\theta-\mathrm{m}=90 \pm 10^{\circ}\right)$

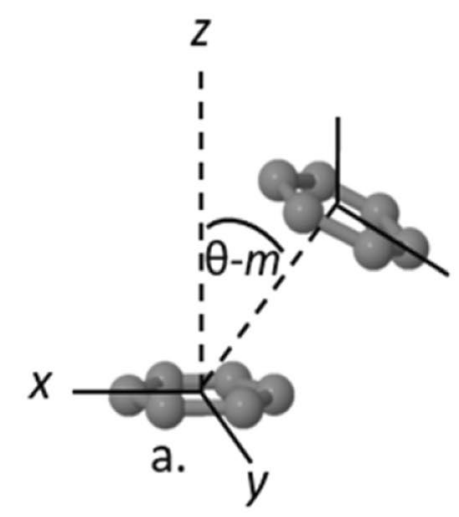

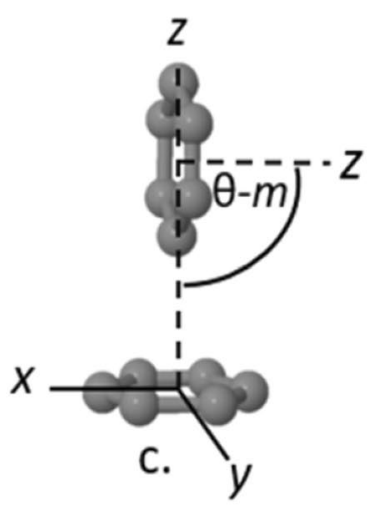

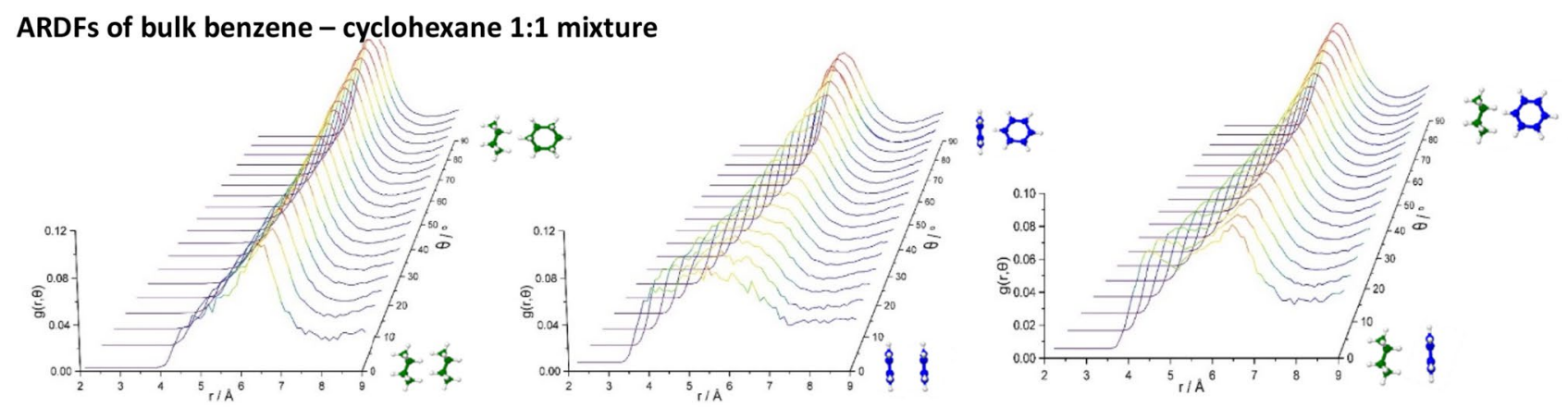

ARDFs of confined flow-1 mixture
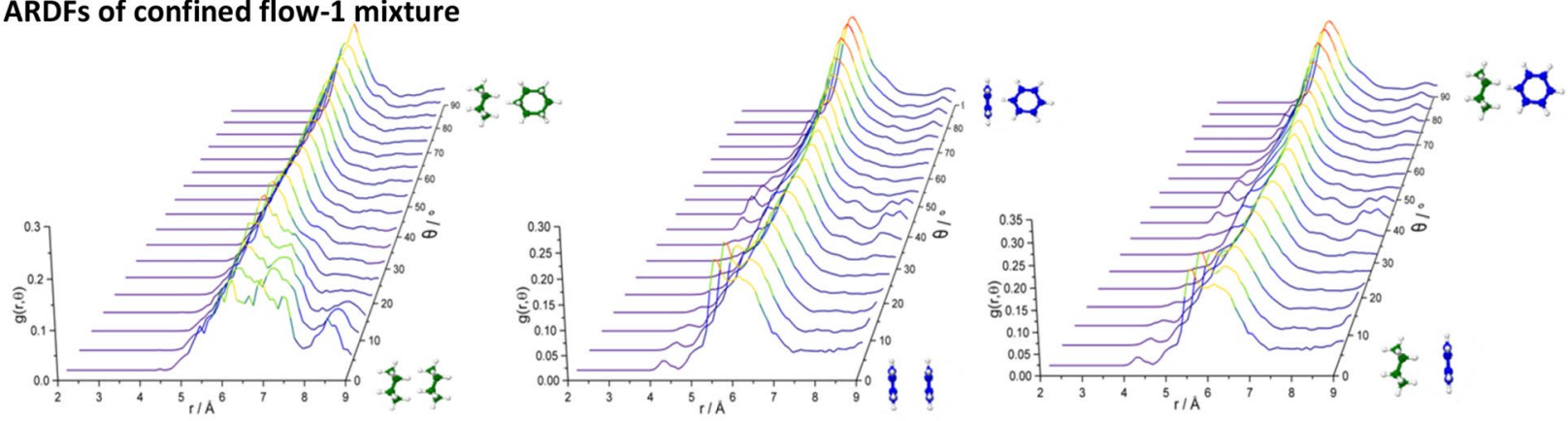

ARDFs of confined flow-2 mixture
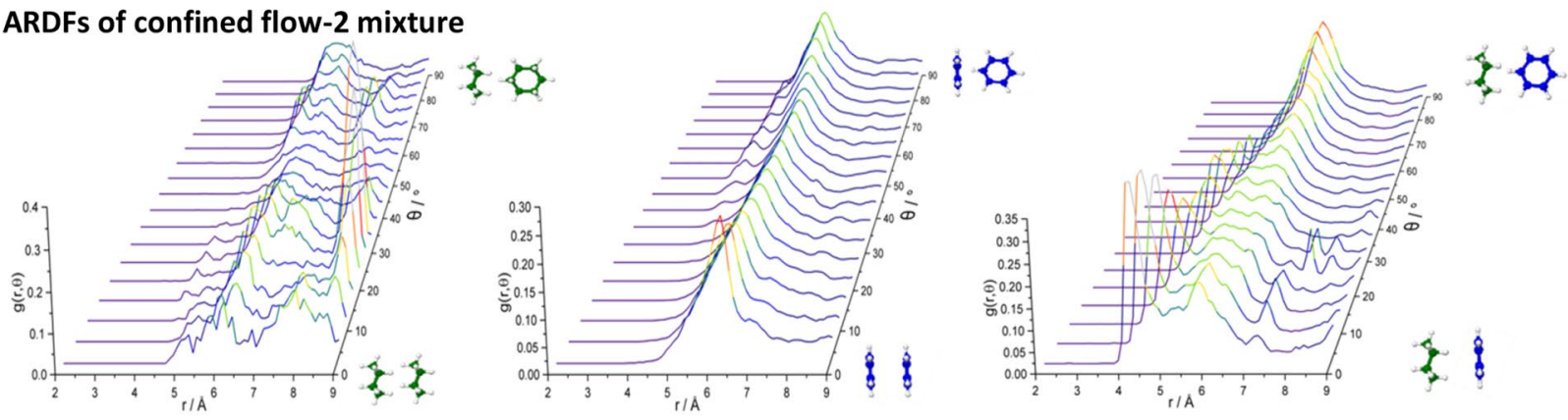

Fig. 6 From left to right, ARDF of like cyclohexane molecules, ARDF of like benzene molecules, ARDF of unlike hydrocarbon molecules in the, from top to bottom, 1:1 bulk mixture, flow 1 and flow
2 systems. Cyclohexane molecules are represented in green, whereas benzene molecules in blue 
molecules at longer distances again. These findings echo those of the equimolar cyclohexane-benzene liquid molecular dynamic simulations by Milano and Müller-Plathe, where the sandwich configuration (parallel) dimer was prevalent at shorter intermolecular distances [17].

Between the two system types, bulk vs confined flow, pronounced differences are observed-mostly within the parallel molecular orientation correlating regions. The bulk liquid mixture like species COM-ARDFs displays a broader region of intensity, 3.5-7.5 $\AA$, than the $4.5-7 \AA$ region in which peak intensity is displayed for flow systems 1 and 2. The lack of intensity at shorter intermolecular distances, indicates a reduced preference for parallel orientations of like molecules in the confined flow systems in comparison to the bulk system. Minimal differences are observed in the perpendicular correlating region, where a singular peak with intensity maxima at $6.0 \AA$ is observed for the flow systems, shifted $0.1 \AA$ from the comparable perpendicular molecular orientation correlating bulk system peaks.

The unlike molecular COM-ARDFs of flow 1 and 2 are comparatively different to one another, as well as to the bulk mixtures. The flow 2 unlike species COM-ARDF at orientational values of $\theta-m=0 \pm 10^{\circ}$ shows a significant peak at intermolecular distances of $4.3 \AA$, indicating a preference for parallel molecular orientations at shorter distances. At the same parallel orientations, a doublet spanning from 4.5 to $7.5 \AA$ is observed in flow 1 , with a very small peak at maximum $4.3 \AA$. The higher cyclohexane content, therefore, increased number of cyclohexane-benzene interactions in the flow 2 system, reasons the significant intensity increase of the $4.3 \AA$ peak. Comparisons of the unlike molecular flow 2 ARDF with the corresponding bulk liquid ARDF shows an increase in parallel oriented unlike molecular interactions in the confined system. This effect of confinement has been previously detailed by Falkowska et al., when studying the structure/orientational changes of neat benzene [7].

\subsection{Spatial Density Functions}

To further probe these interactions, the spatial density functions (SDFs) of the mixtures have been produced. The SDFs display the most probable positions of surrounding molecules in three dimensions from a reference central species. In this work, the SDFs have been produced using the Aten software, written and maintained by Youngs [19].

Figure 7 displays SDFs detailing all molecular interactions within the first coordination shells of the respective central molecule, in the $1: 1$ bulk liquid mixture and in the 1 and 2 flow systems. The top $20 \%$ of all molecules in the coordination shell has been plotted. Only one bulk SDF set is shown as all bulk mixtures studied produced near identical SDFs.

In all bulk liquid mixtures it is observed that like cyclohexane molecules occupy positions perpendicular to the aliphatic carbon-carbon bonds, as well as positions above and below the ring. Six-fold symmetry for like benzene molecules perpendicular to the aromatic carbon-carbon bonds is shown, in addition to density above and below the plane of the ring. The first coordination shell SDFs for unlike species resembles those of the respective central molecules like species arrangements.

The spatial ordering in the confined flow systems is less defined than that shown of the bulk liquids. In general, the first coordination shell spatial ordering resembles those of the respective central molecules bulk ordering, with untidy but strong six-fold symmetry visible in the benzene molecular density correlating regions in both flow systems. The cyclohexane correlating density regions in flow 2 shows

\section{SDFs bulk benzene - cyclohexane}

\section{1:1 mixture}

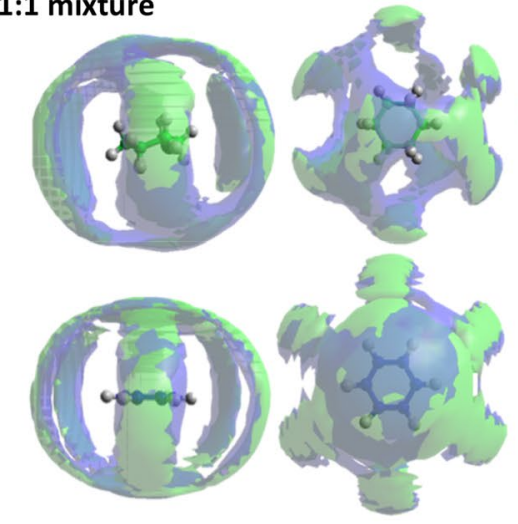

SDFs of confined flow-1 mixture
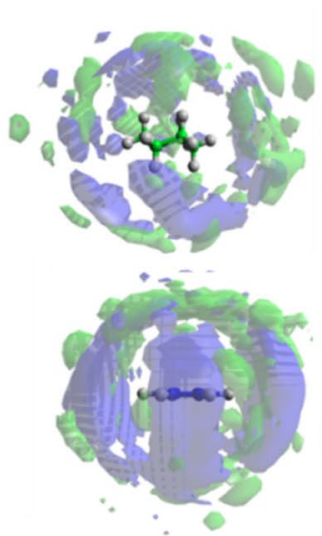

SDFs of confined flow-2 mixture

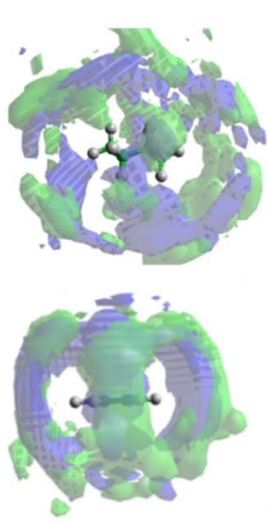

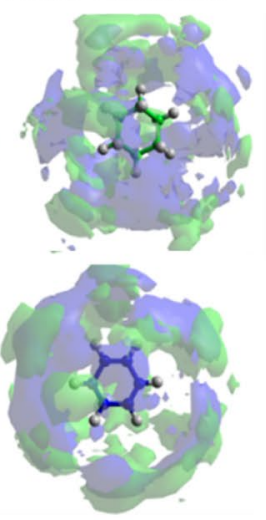

Fig. 7 Top 20\% of molecules found within the first coordination shells of the a 1:1 benzene to cyclohexane bulk mixture, $\mathbf{b}$ the flow 1 confined system and $\mathbf{c}$ the flow 2 confined system. Cyclohexane mol- ecule and cyclohexane corresponding spatial density are represented in green, whereas benzene molecules and benzene corresponding spatial density are represented in blue 
more of a resemblance to those noted in the bulk mixture than is shown by the cyclohexane correlating density regions in flow 1 . The like cyclohexane population of the halo region is diminished in flow 1 in comparison with flow 2 . Both the less defined areas of cyclohexane spatial density and the decrease in halo region spatial density of flow 1 is reasoned by the lower number of cyclohexane molecules present in the system, compared to confined flow system 2 .

Splitting the first coordination shell into two distances, pre and post $4.95 \AA$, and probing the extent of parallel and perpendicular orientations within these distances enables increased understanding of the local ordering in the system. Figures 8 and 9 display the SDFs of parallel molecules (z-axis $\left.\theta-m=0 \pm 10^{\circ}\right)$ and the perpendicular molecules (z-axis $\left.\theta-m=90 \pm 10^{\circ}\right)$, respectively, at distances pre and post $4.95 \AA$, for the $1: 1$ bulk liquid mixture, and flow systems 1 and 2. One bulk SDF set is shown as an example due to the near identical SDFs produced by the bulk mixtures.

Below $4.95 \AA$, in all bulk liquids, parallel molecules reside solely in the halo planes above and below the ring of the central molecule, independent of the central species type. Specifically in the case of benzene, within this distance, there is an absence of like molecules observed directly above or below the centre of the ring. Like molecular spatial density is observed directly above and below the centre of cyclohexane rings. At the same shorter distance range, parallel oriented like and unlike molecular density is found only in the halo region, similar to that observed in the bulk mixtures. However, in the confined flow 1 system, like benzene spatial density is found directly above and below the central ring.

In the bulk mixtures, at distances longer than the $4.95 \AA$ cut-off, like molecular spatial density is observed within the halo plane of the rings, with a clear absence directly above and below the centre of the central molecules. Spatial density is also found in the regions perpendicular to the cyclic carbon-carbon bonds. The location of unlike species spatial density mirrors that of the respective central molecules like species arrangements. In comparison, at distances longer than $4.95 \AA$, the parallel oriented molecular density describing SDFs of flow systems are less populated and less organised than the bulk mixtures. There is minimal parallel oriented cyclohexane spatial density observed in the SDFs of flow 1 . As expected there is slightly more cyclohexane correlating spatial density observed in flow 2 , found in the regions perpendicular to the ring plane with minimal symmetry shown. Parallel benzene oriented molecular SDFs also show lower levels of symmetry, for like benzene and unlike

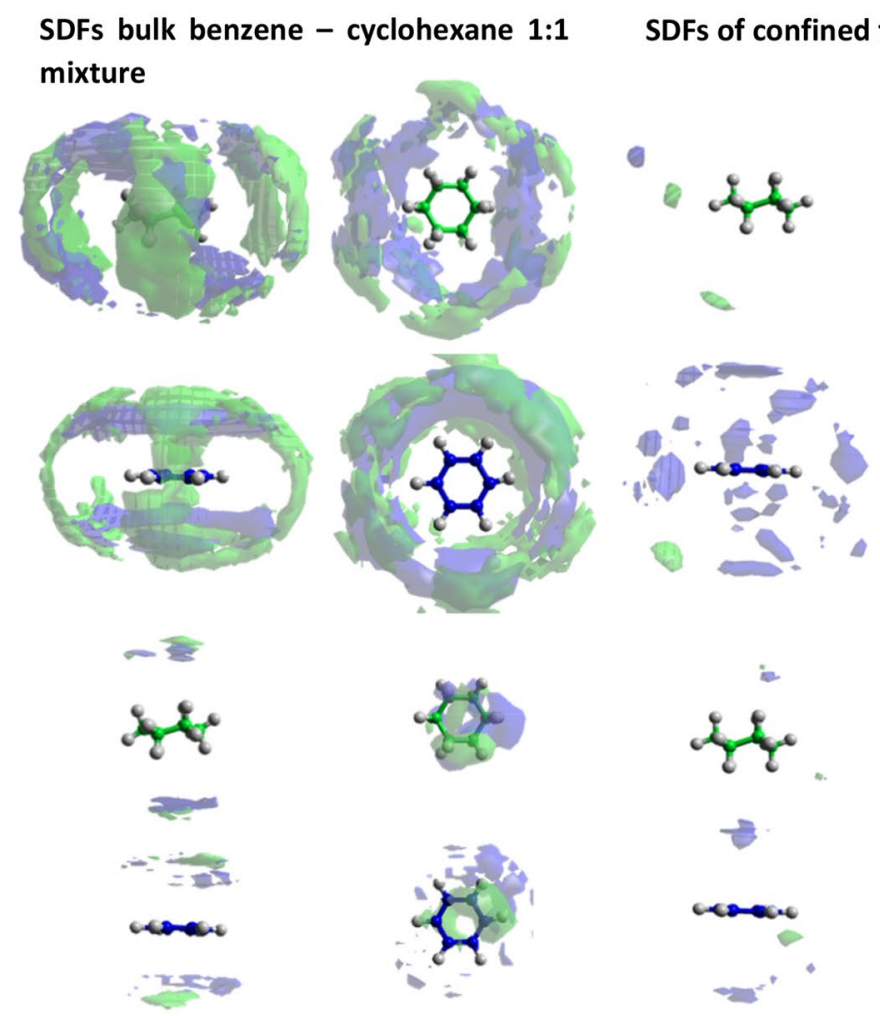

\section{ow-1 mixture}
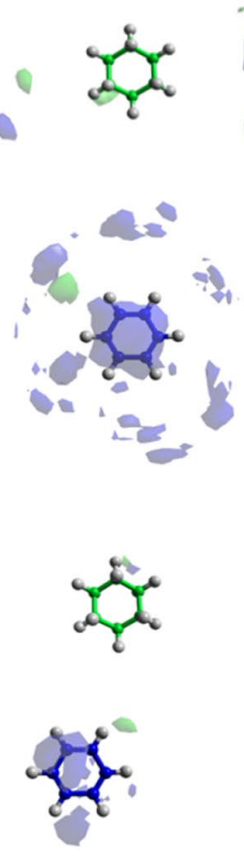

\section{SDFs of confined flow-2 mixture}

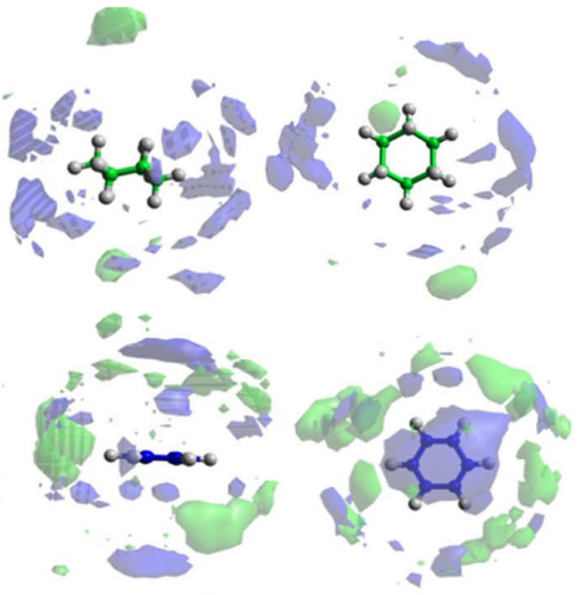

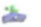
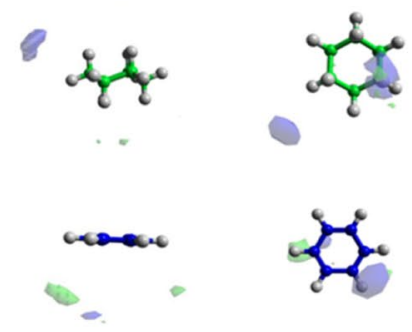

Fig. 8 The spatial density functions of perpendicular molecules ( $z$-axis $\theta=90 \pm 10^{\circ}$ ) within the first coordination shells at distances $r>4.95 \AA$ (top double row) $r<4.95 \AA$ (bottom double row) of the a) $1: 1$ benzene to cyclohexane bulk mixture, b) the flow 1 confined system and c) the flow 2 confined system. Cyclohexane molecule and cyclohexane corresponding spatial density are represented in green, whereas benzene molecules and benzene corresponding spatial density are represented in blue 


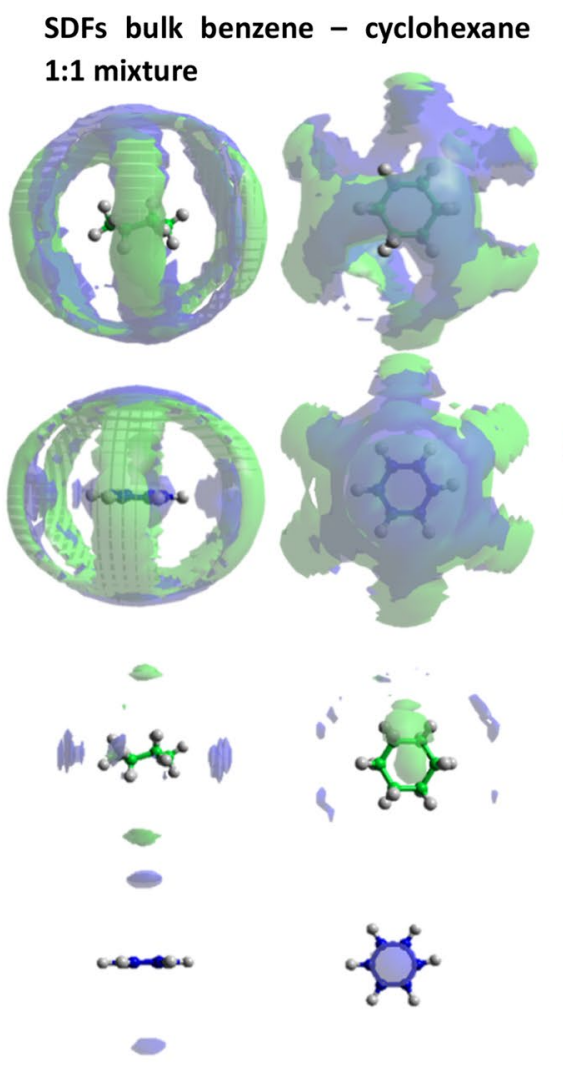

SDFs of confined flow-1 mixture
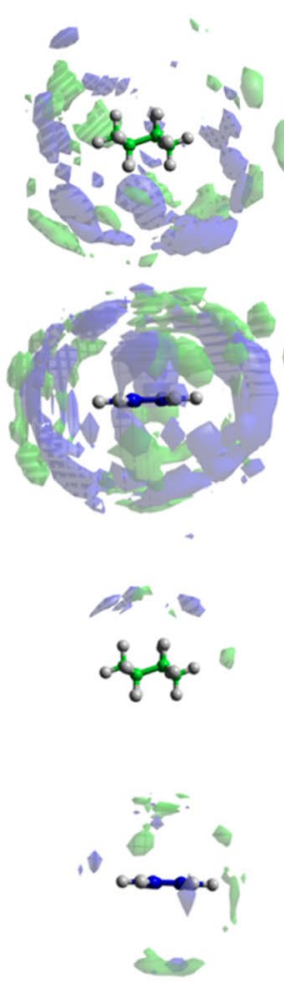

Fig. 9 The spatial density functions of parallel molecules (z-axis $\theta=0 \pm 10^{\circ}$ ) within the first coordination shells at distances $r>4.95 \AA$ (top double row) $\mathrm{r}<4.95 \AA$ (bottom double row) of the a) $1: 1$ benzene to cyclohexane bulk mixture, $\mathbf{b}$ the flow 1 confined system and

molecular interactions. In contrast to that observed in the bulk liquids, like-benzene parallel correlating benzene is observed directly above and below the central ring, as well as in the regions perpendicular to the ring. No change in preference of benzene molecules to occupy the positions in front of the hydrogen atoms of the central ring as opposed to in the middle of the ring forming carbon bonds is found in any of the confined systems, as has been previously observed in the study of neat confined benzene [5]

The bulk mixture SDFs of perpendicular molecules around the benzene central species shows that both like and unlike molecules position directly above and below the plane of the ring. This preference is observed for like cyclohexane perpendicular orientated molecular spatial density, although not as precisely centred above the central molecular. Interestingly, at this short distance, perpendicular orientated benzene molecules are found perpendicular to the carbon-carbon bonds of the central cyclohexane species, instead of showing positional preference within the halo region. Observing perpendicular interactions in the plane of carbon-carbon bonds at such close distances is a phenomenon not previously observed in the Falkowska study of sented in blue
SDFs of confined flow-2 mixture
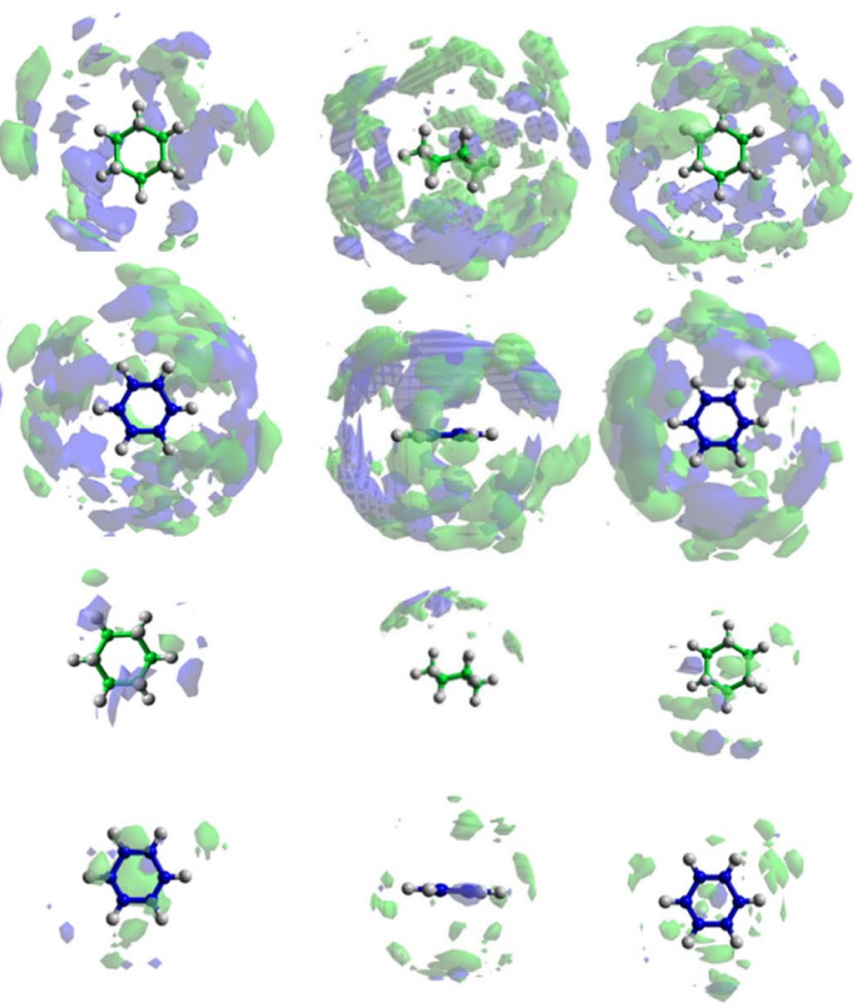

c the flow 2 confined system. Cyclohexane molecule and cyclohexane corresponding spatial density are represented in green, whereas benzene molecules and benzene corresponding spatial density are repre-

neat cyclohexane bulk liquid [20]. The previous comparable study of the benzene bulk liquid observed a slightly shorter distance cut-off, $4.85 \AA$, which similarly showed an absence of spatial density corresponding to perpendicular molecules in the regions perpendicular to the aromatic carbon bonds. It is reasonable to suggest that the mixing of the two hydrocarbon components results in this phenomenon. The more sterically hindered larger cyclohexane perpendicularly oriented molecules occupy the halo region of the central cyclohexane species favourably at this distance. Comparably, perpendicular oriented benzene molecules are less sterically restricted and, therefore, corresponding spatial density is found in the regions perpendicular to the carbon-carbon bonds of the central cyclohexane species. Comparatively less order is displayed in the, short intermolecular distance probing, perpendicular oriented molecular SDFs of the flow mixtures. Noted is a reduced amount of perpendicular like-benzene molecular correlating spatial density, with no positional preference for the regions directly above and below the central ring.

In all bulk mixtures, at first coordination shell length distances longer than $4.95 \AA$, perpendicular orientated molecules occupy the halo regions including the areas directly 
above and below the ring centres, as well the regions perpendicular to the aromatic carbon bonds. As expected, the spatial ordering of perpendicular molecules at this distance resembles that of the respective central molecules like species arrangements with a high level of symmetry shown. At the same longer intermolecular distances, some molecular density symmetry echoing the like species arrangements is observed in the perpendicular oriented molecular describing flow systems SDFs. The six-fold symmetry of like benzene molecular density is more identifiable than any other arrangements. Unlike in the bulk mixtures, not noted in any of the like or unlike perpendicular oriented molecular SDFs is spatial density directly above and below the ring centres. The lack of symmetry noted in the positions of perpendicular or parallel oriented cyclohexane correlating molecular density regions, around either benzene or like-cyclohexane species, is in keeping with the decrease in symmetry found upon confinement of neat cyclohexane by Falkowska [7].

\section{Conclusions}

EPSR has been used to iteratively refine bulk and confined cyclohexane-benzene mixture simulations against TNS data, obtained by use of a novel Flow NeuNMR investigative technique. The fits obtained by the optimised models show good agreement with the experimental total structure factors. The long acquisition time enabled by the use of Flow NeuNMR resulted in improved signal to noise ratio in the experimental data, however, slightly more noise is still present in the flow data versus the bulk data-mostly apparent in the COMRDFs, COM-ARDFs and SDFs.

Minimal differences in the local ordering of molecules in the cyclohexane-benzene bulk hydrocarbon mixtures have been observed, with nearly identical COM-RDFs, COMARDFs and SDFs produced for all bulk systems. Analysis of the molecular compositions within the first co-ordination shells, of all bulk liquid mixtures and confined mixtures, has predictably emulated that of the overall hydrocarbon ratios.

At short intermolecular distances, unlike molecular dimers and like molecular benzene dimers present in the bulk liquids show preference for parallel orientations. For like and unlike species within the first coordination shell, all investigated orientational spatial density plots closely resembled those of the respective central species neat liquid molecular arrangements. The spatial organisation of hydrocarbon molecules within the bulk mixtures mostly echoes that of the pure liquids; however, the presence of perpendicular oriented benzene molecules in the regions perpendicular to the carbon-carbon bonds of the cyclohexane species at short distances is unique to the liquid mixtures.

The molecular interactions in the confined systems have shown altered spatial and orientational preferences in comparison to the bulk mixtures. A lower prevalence of parallel oriented molecules is noted in the confined systems for like dimers at short distances. Whereas, in the confined flow system containing the highest proportion of cyclohexane molecules, an increased preference for the parallel orientations of unlike-molecular dimers at short distances has been found. The spatial organisation of parallel and perpendicular oriented molecules in the confined systems are also altered upon confinement. The preference shown in the bulk liquids for parallel and perpendicular hydrocarbons to occupy the halo regions of the corresponding like and unlike species, at shorter intermolecular distances, is much less pronounced in the confined mixtures. At longer distances the spatial organisation slightly resembles that of the respective central molecules like-species arrangements, with considerably more disorder noted in the confined system. The like benzene molecular spatial organisation is the most altered upon confinement, with parallel oriented molecules rather than perpendicular molecules found directly above and below the central rings at longer first coordination shell distances.

Supplementary Information The online version contains supplementary material available at https://doi.org/10.1007/s11244-021-01437-w.

Funding The authors would like to thank Science and Technology Facilities Council for the allocated beam time at the ISIS Pulsed Neutron and Muon Source (RB1700067), (RB1810608), as well as the funding from EPSRC (EP/N008995/1 and EP/N009304/1, Understanding Liquid Phase Heterogeneous Catalysis to Develop Catalytic Processes).

Data Availability Raw experimental TNS data may be accessed at https://data.isis.stfc.ac.uk/doi/STUDY/103196873, NMR data is available in the supplementary information files.

\section{Declarations}

Conflict of interest There are no conflict to declare.

Open Access This article is licensed under a Creative Commons Attribution 4.0 International License, which permits use, sharing, adaptation, distribution and reproduction in any medium or format, as long as you give appropriate credit to the original author(s) and the source, provide a link to the Creative Commons licence, and indicate if changes were made. The images or other third party material in this article are included in the article's Creative Commons licence, unless indicated otherwise in a credit line to the material. If material is not included in the article's Creative Commons licence and your intended use is not permitted by statutory regulation or exceeds the permitted use, you will need to obtain permission directly from the copyright holder. To view a copy of this licence, visit http://creativecommons.org/licenses/by/4.0/. 


\section{References}

1. Hutchings G, Davidson M, Catlow R, Hardacre C, Turner N, Collier P (2017) Modern Developments in Catalysis, World Scientific, Dordrecht

2. Hansen EW, Sto M, Schmidt R (1996) J Phys Chem 100:2195-2200

3. Knight AW, Kalugin NG, Coker E, Ilgen AG (2019) Sci Rep $8: 1-12$

4. Liu X, Zhang D (2019) J Nat Gas Sci Eng 68:1-21

5. Falkowska M, Bowron DT, Manyar H, Youngs TGA, Hardacre C (2018) Angew Chemie Int Ed 57:4565-4570

6. Abdel Hamid AR, Mhanna R, Lefort R, Ghoufi A, AlbaSimionesco C, Frick B, Morineau D (2016) J. Phys. Chem. C 120:9245-9252

7. Falkowska M, Bowron DT, Manyar HG, Hardacre C, Youngs TGA (2016) ChemPhysChem 17:2043-2055

8. Szala-Bilnik J, Falkowska M, Bowron DT, Hardacre C, Youngs TGA (2017) ChemPhysChem 18:2541-2548

9. Leutzsch M, Falkowska M, Hughes T-L, Sederman AJ, Gladden LF, Mantle MD, Youngs TGA, Bowron D, Manyar H, Hardacre C (2018) Chem Comm 54:10191-10194

10. Youngs TGA, Manyar H, Bowron DT, Gladden LF, Hardacre C (2013) Chem Sci 4:3484
11. Bowron DT, Soper AK, Jones K, Ansell S, Birch S, Norris J, Perrott L, Riedel D, Rhodes NJ, Wakefield SR, Botti A, Ricci MA, Grazzi F, Zoppi M (2010) Rev Sci Instrum 81(033905):1-10

12. Martínez-Edo G, Balmori A, Pontón I, Del Rio AM, SánchezGarcía D (2018) Catalysts 8:617-679

13. Mclain SE, Bowron DT, Hannon AC, Soper AK, Gudrun A computer program developed for analysis of neutron diffraction data, ISIS Facility, Rutherford Appleton Laboratory, Chilton, UK.

14. Soper AK, Bowron DT (2017) Chem Phys Lett 683:529-535

15. Daubert T (1989) Physical and thermodynamic properties of pure chemicals : data compilation. Hemisphere Pub. Corp, New York

16. Chao J, Lin CT, Chung TH (1983) J Phys Chem Ref Data 12:1033-1063

17. Milano G, Müller-Plathe F (2004) J Phys Chem B 108:7415-7423

18. Headen TF, Howard CA, Skipper NT, Wilkinson MA, Bowron DT, Soper AK (2010) J Am Chem Soc 132:5735-5742

19. Youngs TGA (2010) J Comput Chem 31:639-648

20. Falkowska M (2017) Queen's University Belfast.

Publisher's Note Springer Nature remains neutral with regard to jurisdictional claims in published maps and institutional affiliations. 